\title{
Simultaneous transmit and receive polarimetric synthetic aperture radar based on digital beamforming
}

\author{
Hao $\mathrm{Wu}^{\mathrm{a}}$, Dahai Dai \\ College of Electronic Science and Engineering, National University of Defense Technology, 410073 \\ Changsha, China \\ anudtwuhao@163.com
}

Keywords: Digital Beamforming, polarimetric synthetic aperture radar, orthogonal signals, polarization code-diversion mode

Abstract. Synthetic aperture radar is a vital tool for imaging and remote sensing regardless of the villainous weather. Polarimetric synthetic aperture radar obtains the polarization characteristic of targets through polarized wave. This paper presents a novel technique, the combination of digital beamforming and the polarization code-diversion mode, to suppress the range ambiguity of the subapertures based on the simultaneous transmit and reception PolSAR. In the simulation the nulling steering technique proves to be better for the ambiguity mitigation than the conventional radar.

\section{Introduction}

Synthetic aperture radar (SAR), which utilizes the effective long antenna in signal processing instead of the physical antenna, is an effective imaging tool for continuous global coverage[1-2]. Numerous applications of it are e.g., disaster management, traffic observation and controlling, wild area surveillance, and weather forecasting. Nevertheless conventional synthetic aperture radar (SAR) operating with the fixed-polarization antenna is not capable of satisfying the improving demands for wider swath and higher resolution.

Recent developments of synthetic aperture radar (SAR) have led to the generation of Polarimetric Synthetic Aperture Radar (PolSAR), that is, the SAR is capable of the earth's observation and imaging at any possible polarization. Hence such capability permits a more comprehending of the polarization properties of the scatter. Digital beamforming[3] (DBF) is an innovative technique that can be combined with PolSAR for improved performance of remote sensing applications.

Based on the mode of simultaneous transmission and reception, this paper presents PolSAR which employs DBF technique for imaging. The paper is organized as follows. In section 2, we analyse the orthogonal waveforms transmitted by the system. Section 3 establishes the imaging theory of PolSAR which transmits signals and receipts echoes simultaneously. Finally, numerical results are shown in section 4 .

\section{Orthogonal wavefroms of PoISAR}

The linear frequency modulation (LFM) signals with positive and negative slopes are transmitted across two different orthogonal channels. Supposing that LFM wave with positive slope is transmitted by horizontal $(\mathrm{H})$ polarization antenna, while the other through the vertical (V) polarization channel. The basic concept of LFM is illustrated in Fig.1. 


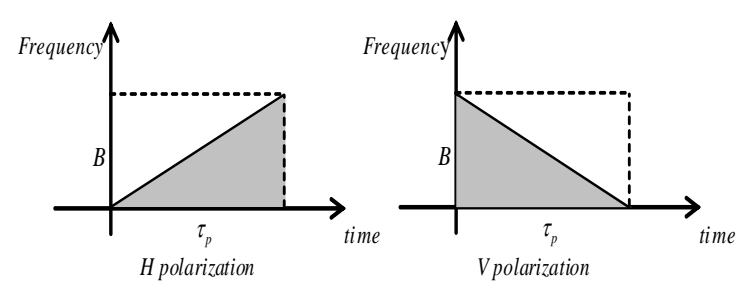

Fig.1 Linear frequency modulation signals transmitted across horizontal and vertical polarization channels.

Transmitted signals $e_{H}(t)$ and $e_{V}(t)$ are given by

$$
\left\{\begin{array}{l}
e_{H}(t)=\frac{1}{\sqrt{\tau_{p}}} \exp \left(\mathrm{j} \pi \gamma t^{2}\right) \operatorname{rect}\left(\frac{t}{\tau_{p}}\right) \\
e_{V}(t)=\frac{1}{\sqrt{\tau_{p}}} \exp \left(-\mathrm{j} \pi \gamma t^{2}\right) \operatorname{rect}\left(\frac{t}{\tau_{p}}\right)
\end{array}\right.
$$

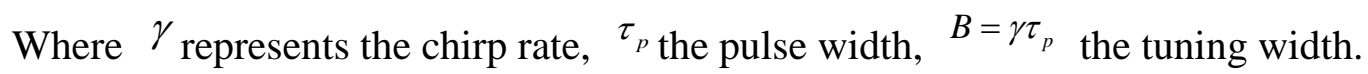

The pulse repetition interval (PRI) of the radar system is $T_{p}$, the velocity $V_{0}$ of the platform should also be considered in calculation . In the period of $n$ PRI:

$$
\begin{gathered}
\varsigma=\tau-\hat{\tau}_{0} \\
\xi=f_{d}-\hat{f}_{d}
\end{gathered}
$$

Where $\tau$ means the time delay of the echo, $\hat{\tau}_{0}$ the estimation value of the delay, $f_{d}$ the Doppler frequency shift, and $\hat{f}_{d}$ represents the estimated value.

$A_{H H}(\cdot, \cdot)$ and $A_{V V}(\cdot, \cdot)$ are the autocorrelation ambiguity functions of $e_{H}(t)$ and $e_{V}(t)$ respectively. $A_{H V}(,$,$) or A_{V H}(,$,$) represents the cross-correlation ambiguity relationship between e_{H}(t)$ and $e_{V}(t)$. They can be expressed by the equations as follows:

$$
\begin{aligned}
& A_{H H}(\varsigma, \xi)=\exp \left(-\mathrm{j} \pi \gamma^{2}\right) \exp \left[\mathrm{j} 2 \pi\left(\xi+\gamma_{\varsigma}\right) \varsigma\right] \frac{\sin \left[\pi\left(\xi+\gamma_{\varsigma}\right)\left(\tau_{p}-|\varsigma|\right)\right]}{\pi\left(\xi+\gamma_{\zeta}\right) \tau_{p}} \\
& A_{V V}(\varsigma, \xi)=\exp \left(j \pi \zeta^{2}\right) \exp \left[\mathrm{j} 2 \pi\left(\xi-\gamma_{\varsigma}\right) \varsigma\right] \frac{\sin \left[\pi\left(\xi-\gamma_{\zeta}\right)\left(\tau_{p}-|\varsigma|\right)\right]}{\pi\left(\xi-\gamma_{\zeta}\right) \tau_{p}} \\
& A_{\text {H }}(\varsigma, \xi)=\exp \left(-\mathrm{j} \pi \gamma \varsigma^{2}\right) \exp \left[-\mathrm{j} 2 \pi \gamma\left(\frac{\varsigma+\xi / \gamma}{2}\right)^{2}\right] \cdot \Pi_{1}(\varsigma, \xi) \\
& A_{H V}(\varsigma, \xi)=\exp \left(\mathrm{j} \pi \gamma \varsigma^{2}\right) \exp \left[-\mathrm{j} 2 \pi \gamma\left(\frac{\varsigma-\xi / \gamma}{2}\right)^{2}\right] \cdot \Pi_{2}(\varsigma, \xi) \\
& \text { Where } \Pi_{1}(\varsigma, \xi)=\frac{1}{\tau_{p}} \int_{\frac{1}{2}\left(-\tau_{p}+|s|-\xi / \gamma\right)}^{\frac{1}{2}\left(\tau_{p}-||-\xi / \gamma\right)} \exp \left(-\mathrm{j} 2 \pi \gamma t^{2}\right) \mathrm{d} t \quad \Pi_{2}(\varsigma, \xi)=\frac{1}{\tau_{p}} \int_{\frac{1}{2}\left(-\tau_{p}+|s|+\xi / \gamma\right)}^{\frac{1}{2}\left(\tau_{p}-|s|+\xi / \gamma\right)} \exp \left(\mathrm{j} 2 \pi \gamma t^{2}\right) \mathrm{d} t \text {. }
\end{aligned}
$$


For the further analysis of the system, the normalized amplitudes of $A_{H H}(\cdot, \cdot), A_{V V}(\cdot, \cdot), A_{H V}(\cdot, \cdot)$ and $A_{V H}(\cdot, \cdot)$ are shown in Fig.2 .

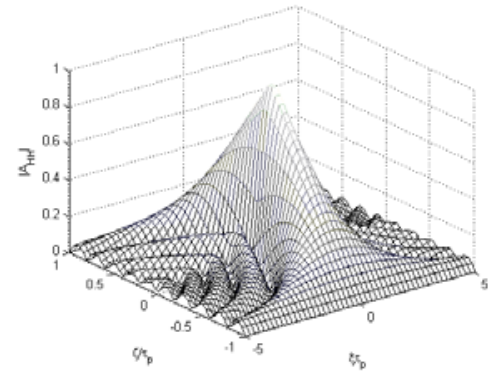

(a) $\mathrm{HH}$

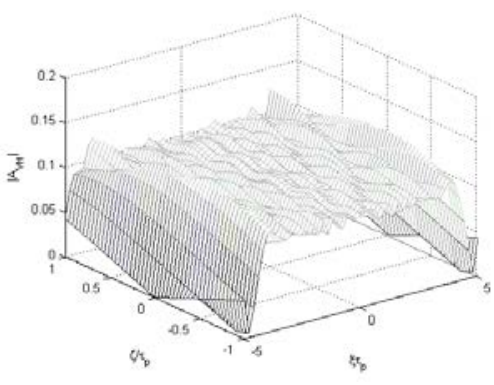

(c) $\mathrm{VH}$

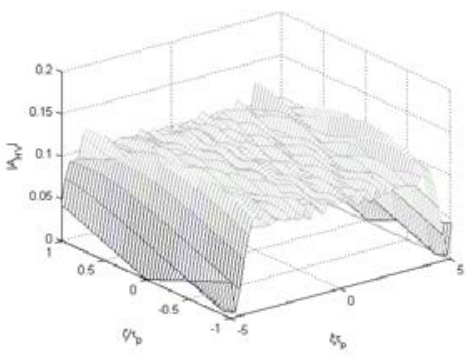

(b) HV

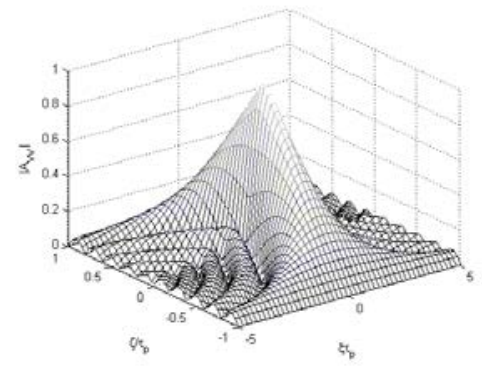

(d) VV

Fig.2 Linear frequency modulation signals transmitted across horizontal and vertical polarization channels.

\section{Imaging theory of simultaneous transmit and reception PolSAR}

\subsection{Digital beamforming}

Wider swath and higher reoulstion are required to meet the increasing demands of radar missions. The single fixed aperture. One of the improming techniques applied in radar imaging is digital beamforming, where the radar has several subapertures. Analog beamforming is circumscribed and restricted, neverthless, digital beamforming ultilizes multiple channels in which received signals are amplified and digitized for further processing. The schemtaic of the digital beamforming is shown in Fig.3 Digital beamforming combines the signals of multiple channels to form beams with adaptive shapes. This technique can be ultilized to suppress the noise in scatter signals, improve the gain of receiving signals in the illumination terrain and get more information about the sactters and suroudings according to the radar echoes.

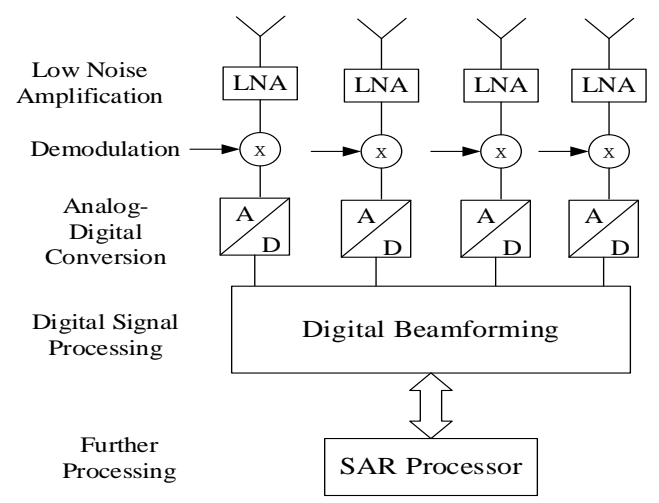

Fig.3 Schematic of Digital beamforming 
Though digital beamforming has been ultilized in the fixed aperture to boost the imaging quality, the improvement may not satisfy the demands for the improved requirements. With subapertures, the SAR system has the properties to realize the high azimuth resoltuion and wide swath. The SAR system records the echoes, ultilizing the large antenna array, to suppress ambiguities and compenste the loss of the beam which is caused by the large terrain illumination to improve the imaging quality.

In this paper, a DBF technique that includes the mulitidimensional waveform encoding on transmit for radar imaging. The subapertures which enable the wide-area imaging with the innovation saptiotemporal modulation of radar pulses.The combination of digital beamforming and spatiotemporal modulation techniques will dramatically improve the performance of the SAR system in the future.Therefore it develops a new approach for remote sensing.

\subsection{Operation modes for PolSAR}

For PolSAR system, high resolution and wide area illumination should be guaranteed. Furthermore, due to the demands of the future tasks, large amount of distinct images are required in within in a short-time frame. Ambiguity is a significant element which shall not be ignored, especially for the PolSAR system.

PolSAR system ultilizes subapertures to transmit and receive signal, due to the limitation of the system, hence a valid operation mode for PolSAR should be chosen to suppress the ambiguity. The operation modes for PolSAR[4] consists of polarization time-diversation(PTD), polarization frequency-diversion(PFD) and polarization code-diversion(PCD).

As illustrated in Fig.4, according to the timing diagram, the antennas firstly transmit a horizontal polarization pulse, then receive the echoes simutaneouly in two different channels.



Fig.4 Timing diagram of the PTD mode

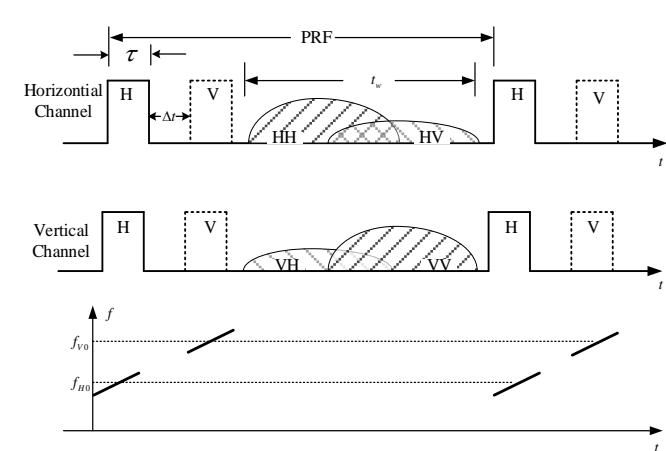

Fig.5 Timing diagram of the PFD mode

PTD mode is common in the PolSAR system and proves to be a successful system. Nevertheless from the diagram we know that the frequncy of combined signals is only half of the pulse repetition frequency(PRF). If we double the PRF to reduce the azimuth ambiguity of the images, however, it may be a bad choice for the range ambiguity. For the large squint angle or high orbit, the standard of image quality is destoryed, even the SAR system is not able to image for remote sensing.

Fig.5 shows the timing diagram of the PTD mode. From the diagram, we know the antenna with two different polarization transimit signals which have differnet central frequency. The horizontal and vertical channels receive the echoes at the same time, through the band-pass filter we can separate signals, then get the polarization information of the scatters. But after the intermediate frequency filter, the channels are complicated and the bandwidth should be doubled. In addition to these disadvantages, the power of the platform is limited which may not satifiy the needs for more 
tramsmitted pairs. Morever when the squint angle is large, the range ambiguity problem may become serious. In Fig 5., $f_{V 0}$ and $f_{H 0}$ represent the different central frequencies of the two channels. The slopes of the chip signals are the same that are transmitted by the two different channels. Therefore by selecting different central frequeies, different polaization signals can be segrated. Separating the copolar and crosspolar signals effectively may be the thorny problem for the intracy of the PolSAR system.

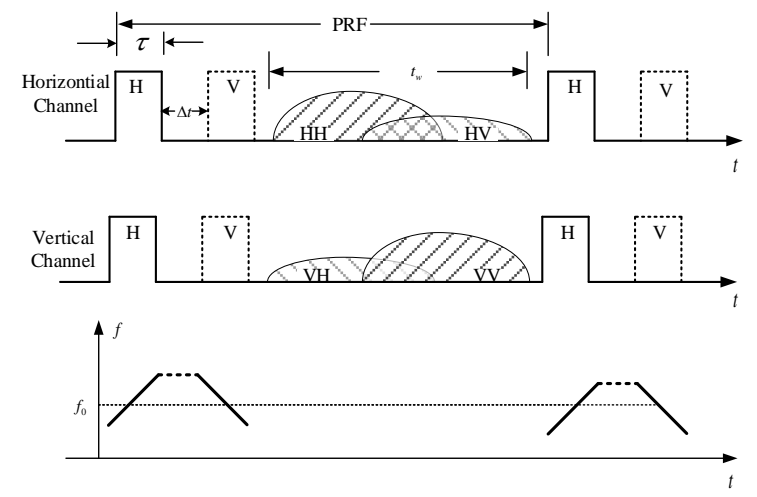

Fig 6. Timing diagram of the PCD mode

As shown in Fig.6 , the PCD mode employes two antennas to tranmit the horizontal and vertical polarization pulses simultaneously that are orthogonally coded. The two polarization antennas receive echoes synchronously and then decode the signals to separate the copolar and crosspolar semaphores. Simlar to the PFD mode, the PRF of multichannel SAR system with PCD mode is identical to that of the fixed polarization SAR. Notwithstanding the PCD mode enables the multi-polarization theoretically, it may not separate the signals that are completely orthogonally. The cross-correlation noise has a certain extent effect on the coded signal which influences the image quality.

With the combination of DBF technique and PCD mode, the PolSAR system will overcome the disadvantages of wide range ambiguities and cross-correlation interference.

\section{Simulation analysis}

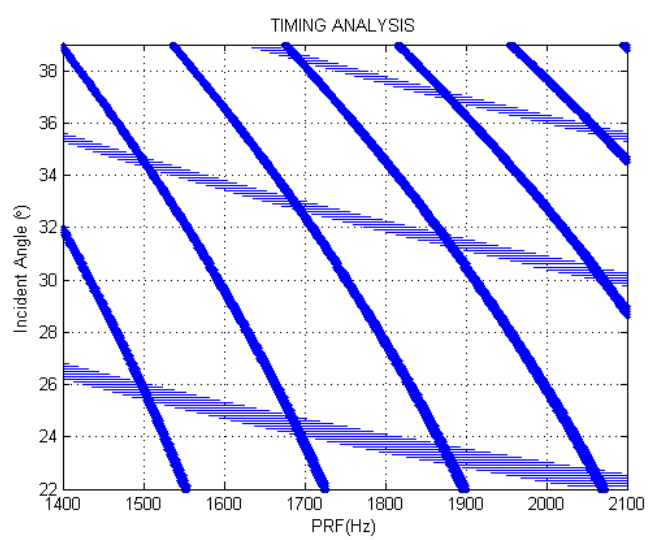

Fig.7 Timing diagram for the PolSAR system

The timing diagram for the PolSAR system is illustrated in Fig. 7, According to the diagram which helps to determine the PRF limit and the range of the incident angle. If the PRF is chosen as $1700 \mathrm{~Hz}$, then incident angle ranges from approximately 24.3 degree to 32 degree. 


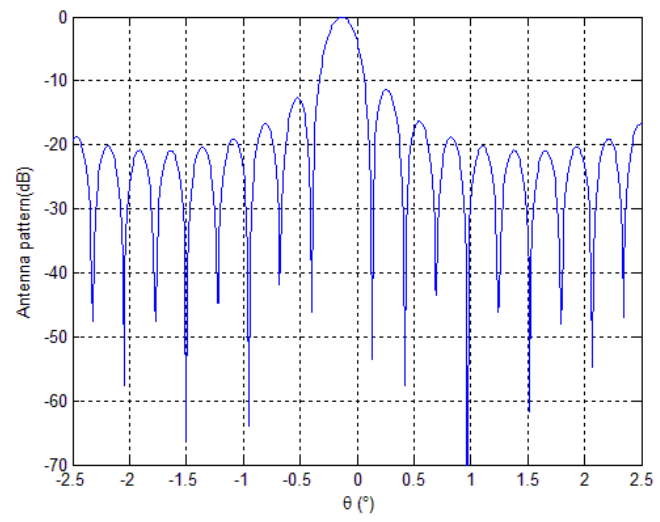

Fig.8 Antenna pattern for the separation of $\mathrm{HH}$ echoes in the range direction

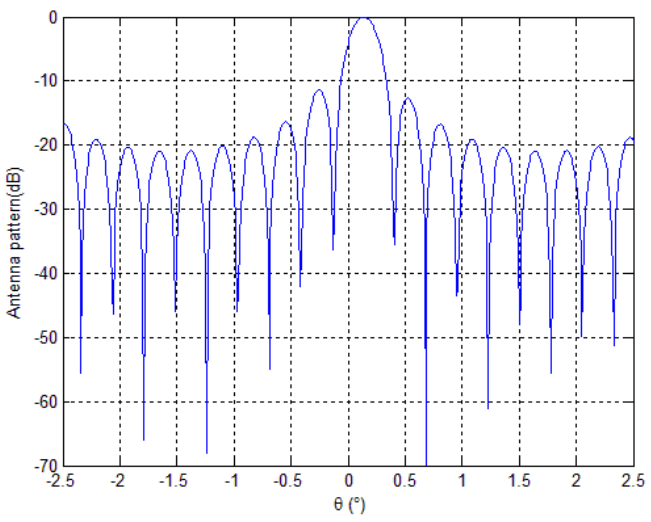

Fig.9 Antenna pattern for the separation of $\mathrm{HV}$ echoes in the range direction

The null steering technique[5] is adopted in the PolSAR system as shown in Fig.8 and Fig.9 to suppress the ambiguity in range and is supposed to separate the echoes primarily in the simulation. Comparing to the methods mentioned(PTD or PFD), the combination of the DBF technology and PCD mode reduces the sidelobe fuzzy and range ambiguity, which will increase the quality of imaging by PolSAR.

\section{Conclusion}

PolSAR contributes to getting the understanding of various target features via polarimetric information and hence plays a vital role in both civil and military. Neverthless the opreation modes of recent PolSAR system all have problems. In this paper, the combination of DBF technique and PCD mode applied on the PolSAR overcomes the weakness of the conventional SAR system. In spite of the complexity of signal processing and complicated load, these disadvantages will be conquered.

\section{Acknowledgements}

This work is sponsoured partly by the National Natural Science Foundation of China (No.61302143, 61501473,61490693) and National High-Tech R\&D Program of China (No.2013AA122202).

\section{Reference}

[1] Cutrona, L. J. "Synthetic aperture radar." Radar handbook 2 (1990): 2333-2346.

[2] Leith, E. N. "Synthetic aperture radar." Optical data processing. Springer Berlin Heidelberg, 1978. 89-117.

[3] Gebert, Nicolas, Gerhard Krieger, and Alberto Moreira. "Digital beamforming on receive: techniques and optimization strategies for high-resolution wide-swath SAR imaging." Aerospace and Electronic Systems, IEEE Transactions on 45.2 (2009): 564-592.

[4] Qi, WeiKong, and WeiDong Yu. "A novel operation mode for spaceborne polarimetric SAR." Science China Information Sciences 54.4 (2011): 884-897.

[5] Mouhamadou, Moctar, Patrick Vaudon, and Mohammed Rammal. "Smart antenna array patterns synthesis: Null steering and multi-user beamforming by phase control." Progress In Electromagnetics Research 60 (2006): 95-106. 